\title{
Structure-based antivirals for emerging and neglected RNA viruses: an emerging field for medicinal chemistry in academia
}

\author{
"Many [neglected viruses] predominantly hit developing countries in tropical and subtropical regions of the world \\ ( $40 \%$ of the world's population are now at risk of contracting dengue fever), but developed countries are by no means \\ immune to their impact."
}

At the recent International Conference on Antiviral Research in San Francisco, CA, USA, 25 years of the use of azidothymidine (AZT) in the treatment of HIV/AIDS were celebrated, along with all the subsequent impressive successes in the therapy of the disease [1]. This is also discussed in the editorial by DeClercq in this issue [2]. Of the oral presentations, $57 \%$ dealt with the discovery and development of antivirals for HIV, hepatitis C, and herpes viruses, all of which lead to chronic infection and the need for prolonged or lifelong therapy [3].

However, there was little room $(8 \%$ of the talks) for emerging viruses and the large number of viruses that are obviously and rightly known as 'neglected viruses.' These include highly pathogenic RNA viruses with a large number of victims, such as measles virus $(>160,000$ child deaths per year), rotavirus $(\sim 500,000-$ 600,000 child deaths every year) or dengue virus ( $\sim 50$ million people infected each year; thousands, again mostly children, die from the haemorrhagic form of dengue fever).

Many of these viruses predominantly hit developing countries in tropical and subtropical regions of the world ( $40 \%$ of the world's population are now at risk of contracting dengue fever), but developed countries are by no means immune to their impact. This is exemplified by West Nile virus, which was introduced from Israel and Egypt into the USA, where it apparently arrived on the East coast in 1999 and became endemic in all 48 contiguous states within 5 years (1356 cases of fever and encephalitis, with 44 deaths, in the USA in 2008). Another example is the global severe acute respiratory syndrome
(SARS) epidemic, which, from its origin in Southern China spread to 29 countries within a few weeks between February and June 2003. While the death toll resulting from the epidemic was relatively low ( $~ 800$ deaths among $\sim 8700$ people infected), the global economic cost was estimated at approximately US \$59 billion. Those who witnessed the deserted streets of Beijing at the peak of the SARS outbreak in May 2003, will never forget the enormous impact on normal daily life caused by the epidemic. Chikungunya virus (CHIKV) is another example of an emerging pathogen. For decades, this virus (which causes high fever and very painful inflammation of the small joints) was associated with relatively small and infrequent outbreaks in West, East and Central Africa. However, in 2005/2006, the virus was apparently dispersed from Eastern Kenya to the islands of the Indian Ocean and subsequently to India (where it had previously made its last appearance in the early 1960s). Just as with dengue virus, CHIKV is spread by the Aedes aegypti mosquito. Following the 2006 outbreak in India, the virus underwent mutations in two surface proteins, thereby facilitating a switch of vector to Aedes albopictus, the Asian Tiger mosquito, which has gradually dispersed from Asia into the Mediterranian region, including Spain, Southern France and Italy. Moreover, the Tiger mosquito has displaced Aedes aegypti in some regions of Central Africa. This is a very worrying trend because the Tiger mosquito is an agressive feeder on humans and is gradually dispersing further afield, with the potential of spreading CHIKV much more widely. The first ever outbreak in Europe occurred in northern Italy in 2007, with more than 200 cases of

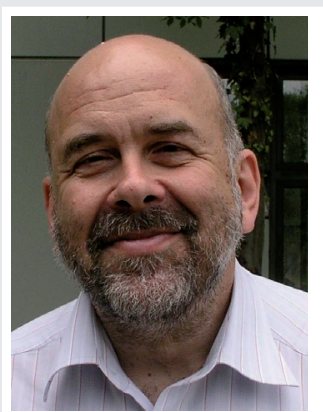

Rolf Hilgenfeld

Institute of Biochemistry, Center for Structural and Cell Biology in Medicine, University of Lübeck, Ratzeburger Allee 160,

23538 Lübeck, Germany Tel.: +49 45I 5004060 Fax: +49 45I 5004068 E-mail: hilgenfeld@ biochem.uni-luebeck.de 
chikungunya fever. This outbreak was traced back to an infected individual traveling from India to Italy. CHIKV continues to cause widespread outbreaks in India and South East Asia. During a recent visit to Pondicherry in southern India, a physician told me that on average, he sees five new chikungunya patients per day in his practice alone.

Despite the recognized emergence of these viruses and the consequent human morbidity and mortality involving millions of individuals, no antiviral agents are available with which to treat the diseases. The major factors for the apparent neglect of these and other viruses are clear: infections with these pathogens are usually self-limiting, either in the vast majority of cases, leading to clearance of the virus by the host's immune system (often in less than 2 weeks) or, in a few cases, the death of the patient.

Consequently, infections by these viruses do not require life-long treatment and, hence, the commercial interest in these widespread diseases is limited. There are a few notable exceptions (for example, Novartis' research program on dengue virus), but the general prospects remain gloomy. Thus, since big pharmaceutical companies are not interested, it is up to scientists in academia and small enterprises ('micropharma') to accept the challenge to commence activities in antiviral drug discovery, with special emphasis on neglected and emerging viruses. While this is a costly venture, some initiatives for new funding schemes are available and will be discussed.

Scientists in academia do not normally have access to the huge chemical libraries that became central to drug discovery via high-throughput screening (HTS) in the pharmaceutical industry during the 1990s. However, as has been emphasized in reviews elsewhere $[4,5]$, there is (once again) a paradigm change going on in industry at present. Rational drug-design approaches, first introduced in the 1980s, are enjoying a well -deserved comeback, with new methodology such as virtual screening and fragmentbased design being added. Individual academic laboratories may still have a hard time raising the funds for implementing the entire range of technologies required for a modern preclinical drugdiscovery pipeline, but consortia of complementary research groups, which may also include small or medium-sized pharmaceutical companies, can certainly cope with this. The advantages of drug discovery in academia have been discussed elsewhere [6].
HIV protease is a good example of what can be at least initiated in an academic environment. The crystal structure of the enzyme was determined in 1989 by the groups of Wlodawer at the National Cancer Center in Frederick, MD, USA and TL Blundell, then at Birkbeck College, London, UK [7,8], in addition to a structural study carried out by Navia at Vertex, Inc. (PA, USA) [9]. It was also in academic groups that the first inhibitors of the enzyme (mostly modified peptides) were designed and co-crystallized with the target [8]. The resulting structures provided the starting point for the pharmaceutical industry, which subsequently synthesized thousands of different inhibitors (PIs) and determined hundreds of crystal structures of their complexes with HIV-1 protease [10]. In some cases, the compounds were derived from inhibitors that had been made in the mid-to-late 1980s against the human aspartic protease renin, which is involved in blood pressure regulation and is distantly related to HIV protease. In any case, none of the nine protease inhibitors introduced into the market starting from 1995 were discovered by HTS; thus, PIs are generally considered to be a prime example of success resulting from structure-based design.

With this encouraging example and the lack of antivirals for large groups of highly pathogenic, neglected RNA viruses in mind, many structural virologists now wish to use the $3 \mathrm{D}$ structures of viral enzymes determined for more than the elucidation of molecular mechanisms. So much more is possible with these results, if they are used for structure-based design of inhibitors targeting these proteins. Of course, structural virologists will either have to expand their repertoire of methods to include virtual screening, molecular modeling, fragment screening and medicinal chemistry, or team up with specialists in these fields. There is ample opportunity for medicinal chemists here, particularly if they are guided by the 3D structures of their macromolecular targets.

Let us look at two examples of neglected viruses, enteroviruses and coronaviruses, where the structure-based design of inhibitors has been successful or is well underway in academia. Although both groups of viruses have singlestranded, positive-sense RNA genomes, they are very different: the enteroviruses are small, nonenveloped icosahedral particles harboring a genome of fewer than 8000 nucleotides; and the coronaviruses are enveloped with the largest RNA genome currently known $(\sim 30,000$ 
bases). Nevertheless, their main proteases, called $3 \mathrm{C}$ and $3 \mathrm{C}$-like protease, respectively, have a number of features in common, making the design of broad-spectrum inhibitors targeting both enzymes a possibility [11]. In general, proteases are useful targets because they are relatively easy to assay, and the initial steps of drug design are usually based on the known cleavage specificities of the enzymes [12]. The difficult step is translating peptidic leads into nonpeptidic inhibitors with the required stability and bioavailability (a novel approach to this challenge is discussed later).

Belonging to the family Picornaviridae ('small RNA viruses'), the genus Enterovirus includes rhinoviruses, a major cause of the common cold in humans, as well as the enteroviruses. Human rhinovirus (HRV) has been and is of interest to several pharmaceutical companies (see elsewhere for a comprehensive review [13]). Rupintrivir (FIgUre I) was designed by Agouron to interfere with the HRV 3C protease [14-16]. Its development was halted in Phase III clinical trials owing to low efficacy in patients [17]. This failure demonstrates that the demands on safety and efficacy are particularly high in case of treatments of viral infections that are not lifethreatening or which cause mild infections lasting only a few days. It is not even clear whether health insurance policies would pay for these drugs, as there is no urgent medical need for them in most patients (although this is different in patients with asthma and chronic obstructive pulmonary disease).

However, there are many relatives of rhinovirus among the enteroviruses that do cause more severe and, sometimes, life-threatening disease. Coxsackieviruses often infect neonates and young children. Most infections are mild or even asymptomatic but, in some cases, patients develop severe conditions, such as encephalitis, meningitis, pancreatitis, myocarditis or acute paralysis [18]. No reliable statistics exist, but it is estimated that several thousand small children succumb to infection by these viruses in Western and Central Europe every year. A causative treatment is not available. This is also true for enterovirus 71 (EV-71), which, like some members of the Coxsackievirus A family, can cause hand, foot and mouth disease (HFMD), a condition that starts with painful blisters on the extremities and the tongue and can proceed to meningoencephalitis or pulmonary edema (which may be fatal) in severe cases. In 2008 and 2009, there were two major outbreaks of HFMD in China,

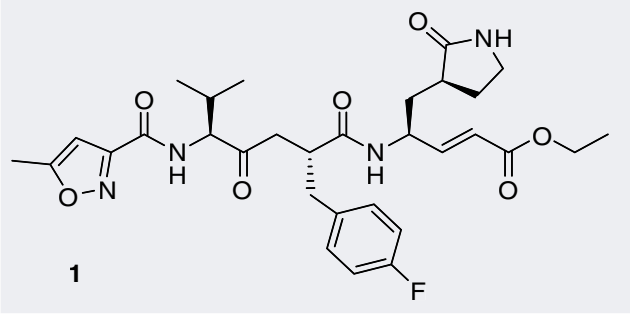

Figure 1. Rupintrivir.

with more than 400,000 people infected during the first outbreak. Relatively few died from the infection but, again, those who did were young children $[19,20]$. EV-71 is believed to have emerged only relatively recently and is now a major global health concern.

Being RNA viruses, the enteroviruses have an RNA-dependent RNA polymerase, which lacks a proofreading function. They therefore exhibit high mutation rates, which is a major driving factor for virus emergence. For this reason, it is difficult to predict which enteroviruses will be important 5 years from now. Consequently, enterovirus inhibitors with broad specificity need to be developed to ensure adequate coverage of the majority of the species in the family. Until recently, there was a lack of structural data on suitable targets for antienteroviral therapy, but this has changed in the past few years, thanks largely to the European Comparative Structural Genomics of Viral Enzymes Involved in Replication (VIZIER) consortium of virologists and structural biologists, who characterized more than 170 RNA viruses and determined approximately 85 crystal structures of key enzymes [21,101]. This included the 3C protease of Coxsackievirus B3 [TAN ET AL. manuscript in Preparation].

The $3 \mathrm{C}$ proteases of these viruses are useful targets for broad-spectrum inhibitors, because they are highly conserved. The amino acid sequences share $40-60 \%$ sequence identity between individual family members, and the $3 \mathrm{D}$ structures are highly similar. This includes the structures of the HRV-14 enzyme [22], as well as the 3C proteases from Coxackievirus B3 [11] [Tan ET AL., Manuscritt in Preparation], EV-68 and EV-71 [Tan ET AL., Unpublished Data]. At their active site, these proteases carry a Cys-His-Glu (or Asp) catalytic triad, with the cysteine offering ample opportunity for covalent modification by nonreversible inhibitors. The common approach to this is to start with a peptide corresponding to the nonprimed half (see elsewhere for nomenclature [23]) of the enzyme's substrates, which are 
the cleavage sites in the viral polyprotein. Next, an electrophilic warhead is attached to the carboxylic end of the P1 residue, to react with the catalytic cysteine of the protease. Carrying an $\alpha, \beta$-nonsaturated alkyl ester moiety, rupintrivir (I; Figure I), as well as many of the experimental inhibitors synthesized in academic laboratories, act as Michael acceptors for the nucleophilic Cys residue [14]. Elaboration of the ester group will address the S1' and S2' pockets of the target enzyme $[24,25]$. Other electrophilic groups that have been used in noncompetitive inhibitors include aldehydes, isatins, homophthalimides and halomethylketones (see elsewhere for review [13]). After modification of their P2 residue, these inhibitors are often not only active against the picornavirus $3 \mathrm{C}$ proteases, but also against coronavirus 3C-like proteases (also known as main proteases, $\mathrm{M}^{\mathrm{pro}}$ ) [26,27]. The most prominent examples of such compounds have been described as inactivators of the SARScoronavirus $\mathrm{M}^{\text {pro }}[11,28,29]$. In addition, benzotriazole esters have been described as efficient modifiers for the SARS-CoV enzyme [30,31].

Peptidic aldehydes are of particular interest, because their binding to the catalytic center of cysteine proteases is reversible [32]. This makes them useful as active site-directing probes. In a process known as 'dynamic ligation screening' (DLS) [33], the covalent adduct between a peptide aldehyde and the target protease, in that case the SARS-CoV $\mathrm{M}^{\text {pro }}$, has been used to screen a small focused library of nonpeptidic nucleophiles, mostly amines, for compounds that would replace the aldehyde (2) from the active site cysteine by formation of a Schiff base (3) and, subsequently an imine (FIgUre 2). This coupling reaction will only work in the presence of the target enzyme, and only with aromatic amines $\left(\mathrm{Ar}-\mathrm{NH}_{2}\right)$; the resulting mixed peptidic/nonpeptidic imine is often a moderately active inhibitor $\left(\mathrm{K}_{\mathrm{i}} \sim 50 \mu \mathrm{M}\right)$. In a second step, the most efficient amine in this reaction is modified to carry an aldehyde moiety itself (4), which in turn is reacted with the catalytic cysteine of the protease, and the same library of nucleophiles is once again screened. This procedure has been used to discover nonpeptidic, competitive inhibitors (5) of the target protease in a very short time, starting from a peptidic aldehyde that was easily designed on the basis of the known subsite specificity of the protease.

While the DLS procedure works both for coronavirus 3C-like proteases and enterovirus $3 \mathrm{C}$ proteases, the former do harbor some special features. A total of 8 years after the first crystal structures of coronavirus $\mathrm{M}^{\text {pro }}$ s were reported [26,27], the enzyme still surprises us. Recently, two articles addressed the question of how the protease can liberate itself from the viral polyprotein, of which it is an integrated part (nonstructural protein 5) before autocleavage. Chen et al. showed that mutations known to destroy the $\mathrm{M}^{\text {pro }}$ dimer and, thereby, inactivate the mature enzyme, still allow autoactivation [34]. Furthermore, Zhang et al. elucidated how this 'premature' enzyme may look in three dimensions. They presented the crystal structure of an $\mathrm{M}^{\text {pro }}$ octamer, carrying an interesting domain swap, which explained the data presented by Chen et al. in structural terms [35]. These new views on a viral protease in its premature state may offer new ideas for the design of inhibitors directed at the maturation process.

(A)

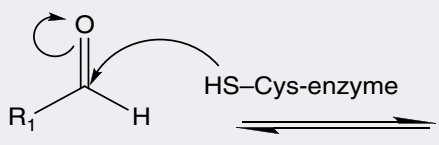

2

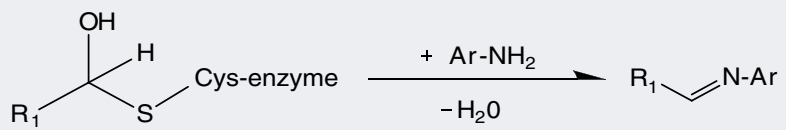

-HS-Cys-enzyme

(B)

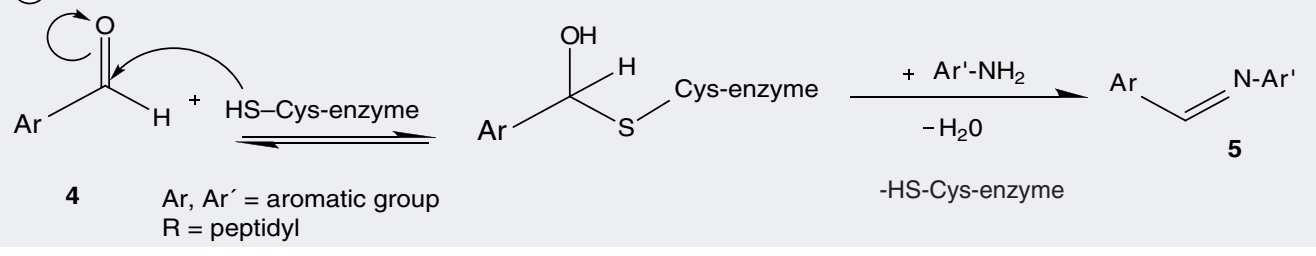

Figure 2. Dynamic ligation screening. 
By no means do I want to suggest that inhibitors of viral cysteine proteases cannot be discovered by screening of a chemical library. On the contrary, the chances of identifying competitive inhibitors that do not modify the active site covalently are much higher with this approach (or with virtual screening) than with structure-based de novo design, which will always be biased by the knowledge of the interaction between the peptidic substrate and the target enzyme. Occasionally, laboratories in academia have their own moderately sized compound libraries, or they have access to libraries established by consortia of academic institutions [36]. For example, by screening a library of approximately 50,000 compounds, a nonpeptidic, small-molecule hit has been discovered as an inhibitor of the second cysteine protease of the SARS virus, the papain-like protease, and optimized to fit the $3 \mathrm{D}$ structure of the enzyme [37].

Of course, one might ask, does it make sense to design and synthesize new SARS$\mathrm{CoV}$ inhibitors, 7 years after the outbreak in 2003? Apart from a few subsequent cases that occurred in China in 2004, the virus has not been detected in humans since. My answer to this question is a definite 'yes'. The virus is almost certainly hiding in its natural reservoir, most likely bats. Coronaviruses of both groups 1 and $2 \mathrm{~b}$ (to which SARS-CoV belongs) have been detected in bats in Southern China as well as in Ghana, Eastern Europe and Northern Europe [38-42]. In various studies, coronaviruses were detected in $5-25 \%$ of the bats investigated. Therefore, zoonotic transmission to humans in the future has to be expected, whether directly or via an intermediate carrier (such as civets in the 2003 SARS outbreak). We must not again be caught by surprise or be almost completely helpless, as we were during the first outbreak. Moreover, since 2003, several new coronaviruses have been discovered, with two of them, HCoV-NL63 and $\mathrm{HCoV} \mathrm{HKU} 1$, being important human pathogens causing infection of the upper respiratory tract $[43,44]$.

As much as the work on inhibitor discovery for the SARS-CoV proteases may bear fruit for future outbreaks of the same or related viruses, the inhibitors that have been designed for enterovirus $3 \mathrm{C}$ proteases may also become relevant for the complete eradication of poliovirus [45]. Through a very successful global vaccination program, this member of the genus
Enterovirus is almost extinct, but it is proving almost impossible to eradicate the virus completely. Poliomyelitis is still endemic in Nigeria, India, Pakistan and Afghanistan [102], and, at present, there is an outbreak in Tajikistan [103]. Furthermore, there is evidence for recombination of the attenuated live vaccination strain with field strains of Coxsackieviruses of the A clade and for immunocompromised people shedding the vaccination strain of the virus $[46,47]$. As a result, the WHO has launched a call for the development of antivirals directed at poliovirus [48]. The good news is that on the basis of structural similarity between the target enzymes, it can be predicted that inhibitors of the 3C protease of other enteroviruses will also inactivate the poliovirus enzyme [49]. Preliminary tests are being carried out at this time.

Having realized the value of their viral protein structures for antiviral drug discovery, a number of research groups from the VIZIER project [101] (which ended in 2009) have teamed up with medicinal chemists and experts in small animal models of diseases caused by RNA viruses, to form a new consortium designated 'small-molecule inhibitor leads versus neglected and emerging RNA viruses' (SILVER), which aims at capitalizing on the structural output of VIZIER and using this large body of information for antiviral drug discovery. The goal of the project is to demonstrate proof of principle for a minimum of two antiviral compounds in a small animal model. Compounds to be developed initially will likely be directed at enteroviruses (including the emerging EV-71) and dengue virus. It is hoped that, if successful, the SILVER project will motivate the pharmaceutical industry to collaborate and perhaps develop some of the lead compounds discovered within the new project. Alternatively, another consortium, the International Consortium on Antivirals (ICAV) has been created by Canadian scientists in response to the SARS outbreak in Toronto, and has since formed a global network of scientists in academia who design and synthesize antivirals [104]. The idea behind ICAV is to identify the best antiviral compounds available within the network and develop them further in preclinical and, ultimately, clinical trials. Such objectives will be staggeringly expensive, but ICAV has developed new funding schemes involving governments, nongovernmental organizations and charities, and has so far been quite successful 
in implementing these. The ambitious goal of the consortium is to provide access to new antivirals at a reasonable cost for the developing countries that need them most, but also to license the compounds to the pharmaceutical industry in developed countries. Clearly, both SILVER and ICAV will need the best available antiviral medicinal chemistry. Hopefully, these academic initiatives will have a positive impact on the current gloomy prospects for antiviral therapies for infections caused by neglected and emerging RNA viruses.

\section{Acknowledgements}

The author thanks Professor EA Gould (Oxford/Marseille) and Dr J Tan, Dr L Zhu and Dr Y Kusov (Lübeck) for discussion.

\section{Financial \& competing interests disclosure}

The author is a member of the three not-for-profit consortia active in antiviral research that have been mentioned in the text: VIZIER, SILVER and ICAV. He has received grants from VIZIER and ICAV in support of his academic research and expects to receive such a grant from SILVER. The author also acknowledges funding for his structural virology and antiviral drug design work from DFG, the European Commission, the Sino-German Center for the Promotion of Research (Beijing), and the Schleswig-Holstein Innovation Fund. The author has no other relevant affiliations or financial involvement with any organization or entity with a financial interest in or financial conflict with the subject matter or materials discussed in the manuscript, apart from those disclosed.

No writing assistance was utlized in the production of this manuscript.

\section{Bibliography}

1 Broder $S$. The development of antiretroviral therapy and its impact on the HIV-1/AIDS pandemic. Antiviral Res. 85, 1-18 (2010).

2 DeClercq E. Antiviral therapy: quo vadis? Future Med. Chem. 2(7), 1049-1053 (2010)

3 Abstracts of the 23rd International Conference on Antivirals, San Francisco, CA, 25-28 April, 2010. Antiviral Res. 86, A1-A76 (2010).

4 Ojima I. Medicinal chemistry at a crossroads: challenges and new possibilities. Future Med. Chem. 1, 401-403 (2009).

5 Raveglia LF, Giardina GAM. Accelarating the drug-discovery process: new tools and technologies available to medicinal chemists. Future Med. Chem. 1(6), 1019-1023 (2009).

6 Wyatt PG. The emerging academic drugdiscovery sector. Future Med. Chem. 1(6), 1013-1017 (2009).

7 Wlodawer A, Miller M, Jaskólski M et al. Conserved folding in retroviral proteases: crystal structure of a synthetic HIV-1 protease. Science 245, 616-621 (1989).

8 Blundell TL, Lapatto R, Wilderspin AF et al. The 3D structure of HIV-1 proteinase and the design of antiviral agents for the treatment of AIDS. Trends Biochem. Sci. 15, 425-430 (1990).

9 Navia MA, Fitzgerald PM, McKeever BM et al. 3D structure of aspartyl protease from human immunodeficiency virus HIV-1. Nature 337, 615-620 (1989).

10 Vondrasek J, Wlodawer A. HIVdb: a database of the structures of human immunodeficiency virus protease. Proteins 49, 429-431 (2002).
11 Lee CC, Kuo CJ, Ko TP et al. Structural basis of inhibition specificities of $3 \mathrm{C}$ and $3 \mathrm{C}$-like proteases by zinc-coordinating and peptidomimetic compounds. J. Biol. Chem. 284, 7646-7655 (2009).

12 Steuber H, Hilgenfeld R. Recent advances in targeting viral proteases for the discovery of novel antivirals. Curr. Top. Med. Chem. 23, 323-345 (2010).

13 De Palma AM, Vliegen I, De Clercq E, Neyts J. Selective inhibitors of picornavirus replication. Med. Res. Rev. 28, 823-884 (2008).

14 Matthews DA, Dragovich PS, Webber SE et al. Structure-assisted design of mechanism-based irreversible inhibitors of human rhinovirus $3 \mathrm{C}$ protease with potent antiviral activity against multiple rhinovirus serotypes. Proc. Natl Acad. Sci. USA 96, 11000-11007 (1999).

15 Johnson TO, Hua Y, Luu HT et al. Structure-based design of a parallel synthetic array directed toward the discovery of irreversible inhibitors of human rhinovirus 3C protease. J. Med. Chem. 45, 2016-2023 (2002).

16 Binford SL, Maldonade F, Brothers MA et al. Conservation of amino acids in human rhinovirus $3 \mathrm{C}$ protease correlates with broadspectrum antiviral activity of rupintrivir, a novel human rhinovirus $3 \mathrm{C}$ protease inhibitor. Antimicrob. Agents Chemother. 49, 619-626 (2005).

17 Patick AK. Rhinovirus chemotherapy. Antiviral Res. 71, 391-396 (2006).

18 Sawyer MH. Enterovirus infections, diagnosis and treatment. Semin. Pediatr. Infect. Dis. 13, 40-47 (2002).
19 Yang F, Ren L, Xiong Z et al. Enterovirus 71 outbreak in the People's Republic of China in 2008. J. Clin. Microbiol. 47, 2351-2352 (2009).

20 Zhang Y, Tan XJ, Wang HY et al. An outbreak of hand, foot, and mouth disease associated with subgenotype $\mathrm{C} 4$ of human enterovirus 71 in Shandong, China. J. Clin. Virol. 44, 262-267 (2009).

21 Coutard B, Gorbalenya AE, Snijder EJ et al. The VIZIER project: preparedness against pathogenic RNA viruses. Antiviral Res. 78, 37-46 (2008).

22 Matthews DA, Smith WW, Ferre RA et al. Structure of human rhinovirus 3C protease reveals a trypsin-like polypeptide fold, RNA-binding site, and means for cleaving precursor polyprotein. Cell 77 , 761-777 (1994).

23 Schechter I, Berger A. On the size of the active site in proteases. I. Papain. Biochem. Biophys. Res. Commun. 27, 157-162 (1967).

24 Lee ES, Lee WG, Yun SH et al. Development of potent inhibitors of the Coxsackievirus 3C protease. Biochem. Biophys. Res. Commun. 358, 7-11 (2007).

25 Kuo CJ, Shie JJ, Fang JM et al. Design, synthesis, and evaluation of 3C protease inhibitors as anti-enterovirus 71 agents. Bioorg. Med. Chem. 16, 7388-7398 (2008).

26 Anand K, Palm GJ, Mesters JR, Siddell SG, Ziebuhr J, Hilgenfeld R. Structure of coronavirus main proteinase reveals combination of a chymotrypsin fold with an extra $\alpha$-helical domain. EMBO J. 21, 3213-3224 (2002). 
27 Anand K, Ziebuhr J, Wadhwani P, Mesters JR, Hilgenfeld R. Coronavirus main proteinase $\left(3 \mathrm{CL}^{\mathrm{pro}}\right)$ structure: basis for design of antiSARS drugs. Science 300, 1763-1767 (2003).

28 Yang $H$, Yang M, Ding Y et al. The crystal structures of severe acute respiratory syndrome virus main protease and its complex with an inhibitor. Proc. Natl Acad. Sci. USA 100, 13190-13195 (2003).

29 Yang H, Xie W, Xue X et al. Design of wide-spectrum inhibitors targeting coronavirus main proteases. PLoS Biol. 3, 1742-1752 (2005).

30 Wu CY, King KY, Kuo CJ et al. Stable benzotriazole esters as mechanism-based inactivators of the severe acute respiratory syndrome 3CL protease. Chem. Biol. 13, 261-268 (2006).

31 Verschueren KHG, Pumpor K, Anemüller S, Chen S, Mesters JR, Hilgenfeld R. A structural view of the inactivation of the SARS-coronavirus main proteinase by benzotriazole esters. Chem. Biol. 15, 597-606 (2008).

32 Al Gharabli SI, Shah ST, Weik S et al. An efficient method for the synthesis of peptide aldehyde libraries employed in the discovery of reversible SARS coronavirus main protease (SARS-CoV M ${ }^{\text {pro }}$ ) inhibitors. ChemBioChem 7, 1048-1055 (2006).

33 Schmidt MF, Isidro-Llobet A, Lisurek M et al. Sensitized detection of inhibitory fragments and iterative development of nonpeptidic protease inhibitors by dynamic ligation screening. Angew. Chem. Int. Ed. Engl. 47, 3275-3278 (2008).

34 Chen S, Jonas F, Shen C, Hilgenfeld R. Liberation of SARS-CoV main protease from the viral polyprotein: $\mathrm{N}$-terminal autocleavage does not depend on the mature dimerization mode. Protein Cell, 1, 59-74 (2010).

35 Zhang S, Zhong N, Xue F et al. 3D domain swapping as a mechanism to lock the active conformation in a super-active octamer of SARS-CoV main protease. Protein Cell, 1 , 371-383 (2010).
36 Lisurek M, Rupp B, Wichard J et al. Design of chemical libraries with potentially bioactive molecules applying a maximum common substructure concept. Mol. Divers. 14, 401-408 (2010).

37 Ratia K, Pegan S, Takayama J et al. A noncovalent class of papain-like protease/ deubiquitinase inhibitors blocks SARS virus replication. Proc. Natl Acad. Sci. USA 105, 16119-16124 (2008).

38 Lau SK, Li KS, Huang Y et al. Ecoepidemiology and complete genome comparison of different strains of severe acute respiratory syndrome-related Rhinolophus bat coronavirus in China reveal bats as a reservoir for acute, self-limiting infection that allows recombination events. J. Virol. 84, 2808-2819 (2010).

39 Yuan J, Hon CC, Li Y et al. Intraspecies diversity of SARS-like coronaviruses in Rhinolophus sinicus and its implications for the origin of SARS coronaviruses in humans. J. Gen. Virol. 91, 1058-1062 (2010).

40 Pfefferle S, Oppong S, Drexler JF et al. Distant relatives of severe acute respiratory syndrome coronavirus and close relatives of human coronavirus 229E in bats, Ghana. Emerg. Infect. Dis. 15, 1377-1384 (2009).

41 Rihtaric D, Hostnik P, Steyer A, Grom J, Toplak I. Identification of SARS-like coronaviruses in horseshoe bats (Rhinolophus hipposideros) in Slovenia. Arch. Virol. 155, 507-514 (2010).

42 Gloza-Rausch F, Ipsen A, Seebens A et al. Detection and prevalence patterns of group I coronaviruses in bats, Northern Germany. Emerg. Infect. Dis. 14, 626-631 (2008).

43 van der Hoek L, Pyrc K, Jebbink MF et al. Identification of a new human coronavirus. Nature Med. 10, 368-373 (2004).

44 Woo PC, Lau SK, Chu CM et al. Characterization and complete genome sequence of a novel coronavirus, coronavirus HKU1, from patients with pneumonia. J. Virol. 79, 884-895 (2005).
45 Collett MS, Neyts J, Modlin JF. A case for developing antiviral drugs against polio. Antiviral Res. 79, 179-187 (2008).

46 Jiang P, Faase JAJ, Toyoda H, Paul A, Wimmer E, Gorbalenya AE. Evidence for emergence of diverse polioviruses from C-cluster Coxsackie A viruses and implications for global poliovirus eradication. Proc. Natl Acad. Sci. USA 104, 9457-9462 (2007).

47 Rakoto-Andrianarivelo M, Guillot $S$, Iber $\mathrm{J}$ et al. Co-circulation and evolution of polioviruses and species $\mathrm{C}$ enteroviruses in a district of Madagascar. PloS Pathogens 3, 1950-1961 (2007).

48 Couzin J. Report concludes polio drugs are needed after disease is eradicated. Science 311, 1539 (2006).

49 De Palma AM, Puerstinger G, Wimmer E et al. Comparative activity of a selected series of antipicornavirus compounds against poliovirus replication in vitro. Emerg. Infect. Dis. 14, 545-551 (2008).

\section{- Websites}

101 VIZIER Project www.vizier-europe.org

102 Global polio eradication initiative. Programme of Work 2009 and financial resource requirements 2009-2013, as of May 2009 WHO, Rotary International, CDC, Unicef. www.polioeradication.org/content/general/ Final_English. GPEIProgrammeofWork2009. pdf

103 World Health Organisation Country Office Tajikistan, WHO Regional Office for Europe, European Centre for Disease Prevention and Control. Outbreak of poliomyelitis in Tajikistan in 2010: risk for importation and impact on polio surveillance in Europe? Eurosurveillance, 15, issue 17, April 29, 2010 www.eurosurveillance.org/Public/Articles/ Archives.aspx

104 International Consortium om Antivirals www.icav-citav.org 